\title{
Two-dimensional transport and wall effects in the thermal diffusion cloud chamber. I. Analysis and operations criteria.
}

Anne Bertelsmann

Richard H. Heist

Fairfield University, rheist@fairfield.edu

Follow this and additional works at: https://digitalcommons.fairfield.edu/engineering-facultypubs Copyright 1997 American Institute of Physics

The final publisher PDF has been archived here with permission from the copyright holder.

\section{Peer Reviewed}

\section{Repository Citation}

Bertelsmann, Anne and Heist, Richard H., "Two-dimensional transport and wall effects in the thermal diffusion cloud chamber. I. Analysis and operations criteria." (1997). Engineering Faculty Publications. 145.

https://digitalcommons.fairfield.edu/engineering-facultypubs/145

\section{Published Citation}

Bertelsmann, A., \& Heist, R. H. (1997). Two-dimensional transport and wall effects in the thermal diffusion cloud chamber. I. Analysis and operations criteria. The Journal of chemical physics, 106(2), 610-623. doi:10.1063/ 1.473400 .

This item has been accepted for inclusion in DigitalCommons@Fairfield by an authorized administrator of DigitalCommons@Fairfield. It is brought to you by DigitalCommons@Fairfield with permission from the rightsholder(s) and is protected by copyright and/or related rights. You are free to use this item in any way that is permitted by the copyright and related rights legislation that applies to your use. For other uses, you need to obtain permission from the rights-holder(s) directly, unless additional rights are indicated by a Creative Commons license in the record and/or on the work itself. For more information, please contact digitalcommons@fairfield.edu. 


\title{
Two-dimensional transport and wall effects in the thermal diffusion cloud chamber. I. Analysis and operations criteria
}

\author{
Anne Bertelsmann and Richard H. Heist ${ }^{\text {a) }}$ \\ Department of Chemical Engineering, University of Rochester, Rochester, New York, 14627-0166
}

(Received 17 June 1996; accepted 2 October 1996)

\begin{abstract}
In this paper we present results of a two-dimensional $(z, r)$ treatment of the mass and energy transfer processes that occur during the operation of a thermal diffusion cloud chamber. The location of the wall is considered in solving the mass and energy transport equations, in addition to the vertical distance, $z$, between the upper and lower plate surfaces. We examine the effect of aspect (diameter to height) ratio on chamber operation; the effects of operation with either a dry or a wet interior chamber wall on temperature, supersaturation, nucleation rate, and total density profiles in the chamber; the effect of overheating the interior of the chamber wall on these conditions within the cloud chamber; and the effects associated with using different density background gases on the operation of the chamber. In a second paper, immediately following, we apply the formalism and the solutions developed in this paper to address the important problem of buoyancy-driven convection that can accompany (seemingly normal) operation of thermal diffusion cloud chambers in nearly all ranges of total pressure and temperature. (c) 1997 American Institute of Physics. [S0021-9606(97)51502-9]
\end{abstract}

\section{INTRODUCTION}

For some time now results of the nucleation experiments reported from our laboratory have indicated that homogeneous nucleation depends both on the amount and the kind of background gas present during the nucleation process. ${ }^{1-7}$ Our reports have generated considerable interest and controversy since (1) it has long been assumed (based upon experimental data) that a noncondensable background or carrier gas present during vapor to liquid homogeneous nucleation acts primarily to maintain the thermal environment and has little, if any, effect on the nucleation process itself; and (2) nearly all experimental nucleation data obtained at lower total pressures and temperatures using expansion-based nucleation devices indicate no (significant) effect of background gas on the nucleation process. ${ }^{8}$

In addition to these experiment-based observations, we note that widely used theoretical models of the nucleation process (e.g., Becker-Döring-Zeldovitch theory and its many derivatives) do not, in general, account explicitly for the presence of a background gas. Furthermore, results of recent nucleation model calculations that examine the dependence of the equilibrium distribution of precritical embryos on the system pressure have been only marginally successful in their attempts to explain the background gas effect. ${ }^{9}$ Also, the effect of total pressure on nucleation rate (through the reversible work of formation of the critical cluster) has been examined utilizing the Nucleation Theorem, but the predicted magnitude of the effect is considerably smaller than observed..$^{10}$ Finally, recent attempts to better understand the (molecular level) interaction between the precritical clusters and the condensable monomer in the presence of a background gas have shown some promise in providing insight

${ }^{a)}$ Author to whom all correspondence should be addressed. into the role of the background gas in establishing precritical cluster embryo distribution. ${ }^{11}$

Portions of the design and several of the operational features of the high-pressure diffusion cloud chamber (HPCC) used for much of our experimental investigations of the background gas effect have lead to concern regarding the quality of the nucleation data obtained with this chamber and conclusions drawn from that data. In spite of the ability of this chamber to reproduce nucleation data in the literature (obtained using more traditional cloud chambers), the small size of this chamber combined with the use of a thick chamber wall (necessary to accommodate high total pressure) and the use of both a wet and a dry interior chamber wall has given rise to reasonable questions regarding the operation of the chamber, the characterization of the chamber conditions, and the overall stability (with respect to convection) of chamber operation during experiments.

Interestingly, similar stability questions regarding the operation of the more traditional version of the TDCC have arisen recently in the context of quantitative nucleation rate measurements carried out at ambient (or below ambient) temperature and total pressure conditions. It has been reported that despite stable upper and lower surface temperatures the measured nucleation rates appear to be seriously affected by relatively small changes in wall temperature ${ }^{12}$ (the interior surface of the TDCC wall is kept dry in these instances through the use of heater wires wrapped around the external surface of the chamber wall). In fact, over the years, we have, on a number of occasions, observed unusual variations in measured nucleation rate apparently related to the (dry) chamber wall conditions. The important point that emerges from a careful review of all these observations and concerns about "proper" TDCC operation is that we have never carefully explored, in a quantitative fashion, the effect of the walls on the interior chamber conditions of the TDCC. 
We have simply assumed that at the center, the walls have no effect provided they are "far enough" away; and our experience suggested that a diameter to height ratio of roughly 5 or greater was sufficient to place the walls "far enough" away. ${ }^{13-15}$

The method of characterizing operating conditions within the TDCC has changed little over the last 25 years. ${ }^{14,15}$ The device is assumed to function (at the center, at least,) in a mode that can be approximated by onedimensional equations describing the transport of energy and mass between the upper and lower plates of the chamber. Solutions to these equations can be as elementary as assuming linear temperature and mole fraction profiles followed by using the ideal gas equation of state to determine the supersaturation profile; ${ }^{16}$ or they can be more detailed, allowing for transport and real gas effects. ${ }^{1-3}$ In all cases, however, the one-dimensional nature of the description has been preserved. In addition to solutions of these one-dimensional equations, virtually everything we know about the "proper" operation and use of the TDCC has been obtained empirically, based entirely upon observation and experience.

In this paper we describe the results of a twodimensional $(z, r)$ treatment of the mass and energy transfer processes that occur during the operation of the TDCC. In this treatment, the location of the wall is considered in the mass and energy transport in addition to the vertical distance, $z$, between the upper and lower plate surfaces. We address specific issues that relate directly to the questions and concerns regarding TDCC operation discussed above. We examine the effect of aspect (diameter to height) ratio on chamber operation. We examine the effects of operation with either a dry or a wet interior chamber wall on temperature, supersaturation, nucleation rate, and total density profiles in the chamber. We examine the effect of overheating the interior of the chamber wall on these conditions within the TDCC. And, we examine the effects associated with using different density background gases on the operation of the chamber. In all cases, in the limit of an infinite aspect ratio, our twodimensional analysis reduces to the standard onedimensional analysis. The two-dimensional model equations are presented and discussed in the next section. The results of our analysis and a discussion of those results are presented in the following section and arranged according to the particular facet of TDCC operation being examined. Our conclusions are summarized in the final section of the paper. In the following paper, we continue our analysis of the twodimensional aspects of TDCC operation by addressing the important issue of stability (with respect to buoyancy-driven convection) of the vapor-gas mixture in the diffusion cloud chamber.

\section{MATHEMATICAL DESCRIPTION}

\section{Transport equations}

\section{Mass balance}

The mass balance for a nonreacting, cylindrically symmetric system can be written in cylindrical coordinates as ${ }^{18}$

$$
\frac{1}{r} \frac{\partial}{\partial r}\left(r N_{r}\right)+\frac{\partial}{\partial z}\left(N_{z}\right)=0,
$$

where $r$ is the radial distance from the center to the wall; $z$ is the vertical distance from the lower to the upper plate; and $N$ is the molar vapor flux in either coordinate. Inside the chamber the vapor is diffusing through a stagnant gas. The flux is given by

$$
\mathbf{N}=c D_{12}\left[\boldsymbol{\nabla} \ln (1-x)-\alpha_{T} x \boldsymbol{\nabla} \ln T\right),
$$

where $c$ is the total molar concentration, $D_{12}$ is the binary diffusion coefficient, $x$ is the mole fraction of the diffusing vapor, $\alpha_{T}$ is the thermal diffusion factor, and $T$ is the temperature. In this expression the first term represents the flux due to a concentration gradient, as derived from Fick's law. The second term expresses the contribution of the Soret or thermodiffusion effect. By substituting the equation for the flux into the mass balance, the balance equation can be solved for the mole fraction profile for a given temperature profile:

$$
\begin{aligned}
\frac{1}{r} \frac{\partial}{\partial r} & {\left[r\left(c D_{12} \frac{\partial \ln (1-x)}{\partial r}-c D_{12} \alpha_{T} x \frac{\partial \ln T}{\partial r}\right)\right] } \\
& +\frac{\partial}{\partial z}\left(c D_{12} \frac{\partial \ln (1-x)}{\partial z}-c D_{12} \alpha_{T} x \frac{\partial \ln T}{\partial z}\right)=0 .
\end{aligned}
$$

As we are interested in more global issues associated with cloud chamber operation and performance, several reasonable simplifications will be made to simplify the solution of this equation. The contribution of the thermodiffusion will be neglected, since the value of $\alpha_{T}$ is generally not well known and the effect of that term is typically small. ${ }^{14,16} \mathrm{It}$ has been suggested, however, that it may play a role in the dependence of the nucleation rate on total pressure. ${ }^{12} \mathrm{We}$ assume the product $c D_{12}$ to be independent of pressure and composition and to depend on temperature in the following fashion:

$$
c D_{12}=\left(c D_{12}\right)_{\mathrm{ref}} \cdot\left(\frac{T}{T_{\mathrm{ref}}}\right)^{b},
$$

where the subscript ref refers to known reference conditions and the exponent $b$ is fitted to experimental data. This relation can be derived for ideal gases, but has also been shown to hold for nonideal systems at low and moderate pressures. ${ }^{18}$ With these simplification the mass balance becomes

$$
\begin{aligned}
\left(\frac{1}{r}+\right. & \left.b \frac{\partial \ln T}{\partial r}\right) \frac{\partial \ln (1-x)}{\partial r}+\frac{\partial^{2} \ln (1-x)}{\partial r^{2}} \\
& +b \frac{\partial \ln T}{\partial z} \frac{\partial \ln (1-x)}{\partial z}+\frac{\partial^{2} \ln (1-x)}{\partial z^{2}}=0
\end{aligned}
$$

This form of the mass balance will be solved together with the energy balance and appropriate boundary conditions to determine the two-dimensional mole fraction and temperature profiles. We note (as an aside-not used in our analysis) that, under certain, special conditions, further simplifications can be made. If the temperature profile is known, the tem- 
perature derivatives can be evaluated; and, thus, the mass balance can be decoupled from the energy balance. If we consider the special case of a linear temperature profile, expressed as ${ }^{16}$

$$
T(z)=T_{L}(1+\beta z / H),
$$

where the temperature of the lower plate, $T_{L}$, has been chosen as the reference temperature, $H$ is the height of the chamber, and $\beta$ is defined by

$$
\beta=\left(T_{U}-T_{L}\right) / T_{L},
$$

and if we further assume that the temperature profile is radially uniform, Eq. (5) simplifies to

$$
\begin{aligned}
& \frac{1}{r} \frac{\partial \ln (1-x)}{\partial r}+\frac{\partial^{2} \ln (1-x)}{\partial r^{2}}+\frac{b \beta / H}{1+\beta z / H} \frac{\partial \ln (1-x)}{\partial z} \\
& +\frac{\partial^{2} \ln (1-x)}{\partial z^{2}}=0 .
\end{aligned}
$$

If the diameter-to-height ratio becomes infinitely large, this equation reduces to the one-dimensional case, and the resulting equation,

$$
\frac{b \beta / H}{1+\beta z / H} \frac{d \ln (1-x)}{d z}+\frac{d^{2} \ln (1-x)}{d z^{2}}=0
$$

can be solved analytically. ${ }^{16}$ The solution for the mole fraction profile is

$$
\ln (1-x)=\frac{(1+\beta z / H)^{1-b} \ln \left[\left(1-x_{L}\right) /\left(1-x_{U}\right)\right]+\ln \left(1-x_{U}\right)-(\beta+1)^{1-b} \ln \left(1-x_{L}\right)}{1-(\beta+1)^{1-b}}
$$

The mass flux is given by

$$
N_{z}=\left(c D_{12}\right)_{\mathrm{ref}} \ln \left(\frac{1-x_{L}}{1-x_{U}}\right) \cdot \frac{(1-b) \beta / H}{1-(1+\beta)^{1-b}} .
$$

\section{Energy balance}

The energy balance for a cylindrically symmetric system with no heat sources or sinks can be expressed in cylindrical coordinates as ${ }^{17}$

$$
\frac{1}{r} \frac{\partial}{\partial r}\left(r q_{r}\right)+\frac{\partial}{\partial z}\left(q_{z}\right)=0,
$$

where $q$ is the energy flux in either coordinate. The energy flux is given as

$$
\mathbf{q}=-\lambda \nabla T+\mathbf{N} \bar{H}+R T \mathbf{N} \alpha_{T}(1-x) .
$$

The first term in Eq. (13) represents the energy flux due to a temperature gradient, as described by Fourier's Law. The thermal conductivity, $\lambda$, is that of the mixture, since both species contribute to the heat conduction. The second term represents the convective energy flux; where $\bar{H}$ is the enthalpy of the vapor. The last term represents the Dufour effect, the energy flux due to a concentration gradient, and $R$ is the gas constant. By substituting Eq. (13) into the energy balance, an equation is obtained that allows the calculation of the temperature inside the chamber for a given mole fraction profile,

$$
\begin{aligned}
\frac{1}{r} \frac{\partial}{\partial r} & {\left[r\left(-\lambda \frac{\partial T}{\partial r}+N_{r} \bar{H}+R T N_{r} \alpha_{T}(1-x)\right)\right] } \\
& +\frac{\partial}{\partial}\left(-\lambda \frac{\partial T}{\partial z}+N_{z} \bar{H}+R T N_{z} \alpha_{T}(1-x)\right)=0 .
\end{aligned}
$$

As described in our discussion of the mass balance, this equation can be simplified by neglecting the Dufour effect.
Since the Soret and Dufour effects are coupled, the argument for neglecting this term is the same as presented earlier. The resulting equation is

$$
\frac{1}{r} \frac{\partial}{\partial r}\left(-r \lambda \frac{\partial T}{\partial r}+r N_{r} \bar{H}\right)+\frac{\partial}{\partial z}\left(-\lambda \frac{\partial T}{\partial z}+N_{z} \bar{H}\right)=0 .
$$

The change in enthalpy can be related to the heat capacity, $c_{p}$, as

$$
\frac{\partial \bar{H}}{\partial r}=\frac{\partial \bar{H}}{\partial T} \frac{\partial T}{\partial r}=c_{p} \frac{\partial T}{\partial r} .
$$

Using this relation, the mass balance [Eq. (1)], and the expression for the mass flux [Eq. 2], the energy balance can be reformulated as

$$
\begin{aligned}
\left(\frac{1}{r}+\right. & \left.\frac{\partial \ln \lambda}{\partial r}-\frac{c D_{12} c_{p}}{\lambda} \frac{\partial \ln (1-x)}{\partial r}\right) \frac{\partial T}{\partial r}+\frac{\partial^{2} T}{\partial r^{2}} \\
& +\left(\frac{\partial \ln \lambda}{\partial z}-\frac{c D_{12} c_{p}}{\lambda} \frac{\partial \ln (1-x)}{\partial z}\right) \frac{\partial T}{\partial z}+\frac{\partial^{2} T}{\partial z^{2}}=0 .
\end{aligned}
$$

This equation is used in conjunction with the mass balance [Eq. (5)] and appropriate boundary conditions to deter- 
mine the two-dimensional temperature and mole fraction profiles in the cloud chamber.

We note (as an aside-not used in our analysis) that, although the dependence of the thermal conductivity on location is complicated since both the temperature and the composition vary from one position to the next, the actual variation in $\lambda$ is fairly small. If one assumes a constant thermal conductivity, $\bar{\lambda}$, Eq. (17) is further simplified while introducing only a small error. In the sample calculation carried out with variable and constant values for the thermal conductivity and for the heat capacity, the temperature profile in the center of the chamber varied, at most, by one or two degrees, while the mole fraction profiles for both calculations were identical. The radial profiles of the temperature and mole fraction were even less affected, since the radial mole fraction and temperature gradients are generally much smaller than the axial ones. Throughout most of the chamber (except in close proximity to the wall) the radial temperature and mole fraction profiles are essentially uniform and the radial gradient of the thermal conductivity is nearly zero. With constant values for the thermal conductivity and the heat capacity (which also varies slowly), the energy balance reduces to

$$
\begin{gathered}
\left(\frac{1}{r}-\frac{c D_{A B} \overline{c_{p}}}{\bar{\lambda}} \frac{\partial \ln (1-x)}{\partial r}\right) \frac{\partial T}{\partial r}+\frac{\partial^{2} T}{\partial r^{2}} \\
-\frac{c D_{A B} \overline{c_{p}}}{\bar{\lambda}} \frac{\partial \ln (1-x)}{\partial z} \frac{\partial T}{\partial z}+\frac{\partial^{2} T}{\partial z^{2}}=0 .
\end{gathered}
$$

We note also that, under special circumstances (but, again, not used here), this equation could be further simplified. The ratio of thermal conductivity to diffusivity and heat capacity is dimensionless and is referred to as the Lewis number (Le). It represents a measure of the relative importance of the conductive to the convective fluxes. Large values of the Lewis number signify that convective transport is negligible compared to conductive transport. If the convective heat flux is neglected, we have

$$
\frac{c D_{12} c_{p}}{\lambda}=\frac{1}{\mathrm{Le}}=0 .
$$

For this special case, the energy balance further simplifies to

$$
\frac{1}{r} \frac{\partial T}{\partial r}+\frac{\partial^{2} T}{\partial r^{2}}+\frac{\partial^{2} T}{\partial z^{2}}=0
$$

If the diameter to height ratio becomes infinitely large, this equation reduces to the simple expression

$$
\frac{d^{2} T}{d z^{2}}=0
$$

The solution to this equation yields the linear temperature profile, which is, on occasion, assumed a priori in simplified calculations. This analysis clearly shows the assumptions involved when using a linear temperature profile.

Once the mass and energy balances [Eqs. (5) and (17)] are solved in conjunction with appropriate boundary condi- tions, the mole fraction and temperature profiles, the density, supersaturation, and nucleation rate at every point in the chamber can be calculated. For the thermodynamic calculations and for calculations of the supersaturation in this investigation we assume ideal gas behavior for convenience (the Poynting correction is included). Nucleation rates are obtained using the Becker-Döring-Zeldovich (BDZ) rate expression.

\section{Boundary conditions}

The proper boundary conditions at the chamber wall for the mass and the energy balances reflect the operating conditions of the chamber (e.g., wet or dry chamber wall). The chamber wall is a wettable surface; and, as such, the supersaturation at this surface cannot be larger than unity. There are two ways in which the supersaturation can be reduced to unity (or below) at the wall.

\section{Dry wall}

In the case of a dry wall the equilibrium vapor pressure is increased until it becomes equal to or larger than the partial pressure of the condensable at the wall. This is achieved by raising the temperature at the wall through heating of the wall. Thus, the minimum temperature at the wall (for unit supersaturation) is given as a function of the mole fraction, $x$; as

$$
T(z, R)=\gamma(z) \cdot T^{\mathrm{eq}}\left(p_{\mathrm{vap}}\right),
$$

where $p_{\text {vap }}$ represents the partial pressure of the condensable adjacent to the wall and is computed as the product of the mole fraction and the total pressure. $T^{\mathrm{eq}}\left(p_{\text {vap }}\right)$ signifies that the temperature is obtained using the vapor pressure equation and the partial pressure of the condensable at a particular value of $z$. The function $\chi(z)$ in this expression is used to allow for overheating at the wall. The lowest possible temperature for which the wall is dry is obtained for a value of $\gamma=1$, for which the supersaturation at the wall is unity. The dependence of $\gamma$ on $z$ indicates that overheating does not need to be uniform, but can be modeled to represent the presence of heating wires. The formulation of the boundary condition at the wall closely mirrors the experimental procedure. In an actual experiment, the wall temperature would be increased (generally by increasing the power to the wall heater wires) until the walls are just dry (to the eye), then reduced slightly to avoid unnecessary overheating. The point at which the wall becomes dry corresponds to the condition that the vapor pressure at each height is equal to or greater than the actual partial pressure of the condensable. For uniform heating $\gamma=1$ yields a best case situation, one in which the temperature disturbance is the smallest possible and still achieves the desired effect. In most experiments, however, the wall is heated by several heating wires, and it is difficult to adjust the temperature at different heights independently. Also, the spaces between the wires may be at temperatures differing from those adjacent to the heater wires. Thus, overheating can occur over some regions of the wall. As a result, the temperature will be elevated beyond the minimum required to clear the wall at certain elevations which will result 
in supersaturations of less than unity at certain locations along the wall. This situation can be simulated by choosing values of $\gamma$ to be greater than unity and a function of height.

For the mass balance, the boundary condition at the wall follows from the assumption that no vapor is condensing there. Thus, the net radial mass flux at the wall has to vanish:

$$
\left.\frac{\partial \ln (1-x)}{\partial r}\right|_{r=R}=0
$$

\section{Wet wall}

In the case of a wet wall, no heat is supplied to the walls. The vapor adjacent to the wall condenses, forming a film on the wall. The mole fraction at the wall will be lowered until the partial pressure is equal to the equilibrium vapor pressure, giving rise to a supersaturation of unity at the wall.

The mole fraction at the wall is given by a phase equilibrium calculation using the temperature at the wall.

$$
x(z, R)=\frac{p^{\mathrm{eq}}(T)}{p_{\mathrm{tot}}},
$$

where $p^{\mathrm{eq}}(T)$ is the equilibrium vapor pressure and is a function of the temperature at the wall, $T(z, R)$. The temperature at the wall cannot be determined a priori. The condensing vapor releases heat that elevates the temperature of the wall above that at the center of the chamber and the condensate film flowing downward from the upper plate slightly cools the wall. The wall temperature further depends on the temperature of the external surroundings relative to that of the chamber, as well as the thickness and thermal conductivity of the cloud chamber ring. In our calculation, we assume that the temperature at the wall corresponds to the temperature within the chamber, so that no temperature gradient exists close to the wall:

$$
\left.\frac{\partial T}{\partial r}\right|_{r=R}=0 .
$$

This assumption, most probably, results in a lower value for the wall temperature and thus a lower equilibrium pressure, giving rise to a larger disturbance in the mole fraction profile. This assumption is analogous to that of the dry wall case, in which the mole fraction profile remains undisturbed, and only the temperature profile is adjusted to prevent condensation. In actual experiments the wall temperature may differ due to the heat released on condensation or differences in the temperature of the external surroundings (especially with thin chamber walls). However, in the absence of detailed knowledge of the wall temperature, we adopt the worst case. If the wall temperature is elevated, the conditions inside the chamber will be an intermediate to the wet and dry wall solutions, since close to the wall both the temperature and the mole fraction profiles are disturbed.

Condensate Film: The reason the interior wall of the TDCC is wet is due to the condensate returning from the upper plate to the lower plate and to vapor from the chamber interior condensing on the wall. The drainage from the upper plate, however, usually occurs as a few localized rivulets and thus may not contribute significantly to the thickness of the film between these rivulets. The film of the condensate on the wall will drain down the wall to the pool on the lower plate. We establish the boundary condition at the wall to be the temperature or the mole fraction at a value of $r=R$, where $R$ is the inner radius of the TDCC ring. There is a thickness associated with the condensate film that can be estimated and shown to be small. We present the analysis in the Appendix. The thickness of the condensate films typical for our experiments is $0.1 \mathrm{~mm}$ or less. The velocity with which the film flows down the interior wall is also of interest. There are important buoyancy-driven convection issues associated with the vapor-gas mixture in the immediate vicinity of the interior wall, and a flowing film may have an effect on that mixture adjacent to the wall. The film falling velocity analysis is also presented in the Appendix. Typical falling velocities for our liquid films were of the order of $1-2$ $\mathrm{mm} / \mathrm{s}$.

The remaining six boundary conditions are identical for the wet and the dry wall case. The temperatures at the top and bottom surfaces are directly amenable to measurement and are known for any given experiment:

$$
\begin{aligned}
& T(0, r)=T_{L}, \\
& T(H, r)=T_{U} .
\end{aligned}
$$

It is assumed that the vapor adjacent to the top and bottom surface is in equilibrium with the liquid film at the top or the liquid pool at the bottom. Thus, the mole fraction can be obtained as

$$
\begin{aligned}
& x(0, r)=p^{\mathrm{eq}}\left(T_{L}, p_{\text {tot }}\right) / p_{\text {tot }}, \\
& x(H, r)=p_{\text {eq }}\left(T_{U}, p_{\text {tot }}\right) / p_{\text {tot }} .
\end{aligned}
$$

The final two boundary conditions required for the solution of the mass and energy balances are those of symmetry at the center of chamber. Here, all radial fluxes vanish, resulting in vanishing radial gradients for the temperature and the mole fraction:

$$
\begin{aligned}
& \left.\frac{\partial T}{\partial r}\right|_{r=0}=0, \\
& \left.\frac{\partial \ln (1-x)}{\partial r}\right|_{r=0}=0 .
\end{aligned}
$$

Solving the mass and energy balances [Eqs. (5) and (17)] using these boundary conditions yields the two-dimensional mole fraction and temperature profiles inside the diffusion cloud chamber. Once these profiles are known, the supersaturation and the nucleation rate can be calculated at every point in the chamber. These solutions being subject to our assumptions of ideal gas behavior, no thermophoretic effects, and Eq. (4).

\section{NUMERICAL SOLUTION}

The partial differential equations presented in the previous section were solved numerically using the method of finite differences. For convenience, the mass and energy 
equations, as well as the boundary conditions, were converted to dimensionless form. The dimensionless variables and parameters are defined by

$$
\eta=\frac{r}{R}, \quad \xi=\frac{z}{H}, \quad \theta=\frac{T}{T^{o}}, \quad \Psi=\ln (1-x) .
$$

The temperature of the lower plate was chosen as reference temperature $T^{\circ}$. The dimensionless mass and energy balances are, respectively,

$$
\begin{aligned}
& \left(\frac{H}{R}\right)^{2}\left[\left(\frac{1}{\eta}+\frac{b}{\theta} \frac{\partial \theta}{\partial \eta}\right) \frac{\partial \Psi}{\partial \eta}+\frac{\partial^{2} \Psi}{\partial \eta^{2}}\right]+\frac{b}{\theta} \frac{\partial \theta}{\partial \xi} \frac{\partial \Psi}{\partial \xi}+\frac{\partial^{2} \Psi}{\partial \xi^{2}}=0 \\
& \left(\frac{H}{R}\right)^{2}\left[\left(\frac{1}{\eta}+\frac{1}{\lambda} \frac{\partial \lambda}{\partial \eta}-\frac{1}{\mathrm{Le}} \frac{\partial \Psi}{\partial \eta}\right) \frac{\partial \theta}{\partial \eta}+\frac{\partial^{2} \theta}{\partial \eta^{2}}\right] \\
& +\left(\frac{1}{\lambda} \frac{\partial \lambda}{\partial \xi}-\frac{1}{\mathrm{Le}} \frac{\partial \Psi}{\partial \xi}\right) \frac{\partial \theta}{\partial \xi}+\frac{\partial^{2} \theta}{\partial \xi^{2}}=0 .
\end{aligned}
$$

The dimensionless boundary conditions are

$$
\begin{array}{ll}
\theta(\xi=0)=1, & \Psi(\xi=0)=\ln \left[1-x^{\mathrm{eq}}\left(T_{L}\right)\right], \\
\theta(\xi=1)=T_{U} / T_{L}, & \Psi(\xi=1)=\ln \left[1-x^{\mathrm{eq}}\left(T_{U}\right)\right], \\
\left.\frac{\partial \theta}{\partial \eta}\right|_{\eta=0}=0, & \left.\frac{\partial \Psi}{\partial \eta}\right|_{\eta=0}=0 ;
\end{array}
$$

dry wall:

$$
\theta(\eta=1)=\gamma \theta^{\mathrm{eq}}\left(\Psi_{\eta=1}\right),\left.\quad \frac{\partial \Psi}{\partial \eta}\right|_{\eta=1}=0
$$

wet wall:

$$
\left.\frac{\partial \theta}{\partial \eta}\right|_{\eta=1}=0, \quad \Psi(\eta=1)=\Psi^{\mathrm{eq}}\left(\theta_{n=1}\right) .
$$

By setting the parameter $(H / R)$ to zero in Eqs. (31) and (32), we obtain the usual one-dimensional solution. In the one-dimensional case the computed results for both the wet and dry wall agree; which is expected since the two cases differ only in their radial boundary conditions. This onedimensional solution has also been compared to solutions obtained from previous analyses of our TDCC and HPCC (high-pressure diffusion cloud chamber) experiments. In those analyses we assumed one-dimensional transport but included nonideal gas behavior. The results for these temperature and mole fraction profiles agree well with those computed using these simplified equations. This provides additional a posteori justification for our simplifications.

Furthermore, by setting the parameter $(1 / \mathrm{Le})$ to zero, the convective energy flux is neglected. If, in addition, the onedimensional case is considered, the temperature profile is found to be linear and the resulting mole fraction profile corresponds to the analytical solution given in Eq. (10), as expected.

The mass and the energy balances are solved simultaneously, since they form a system of coupled partial differential equations. In actuality, the solution is achieved iteratively. In the case of a dry wall where the radial mole

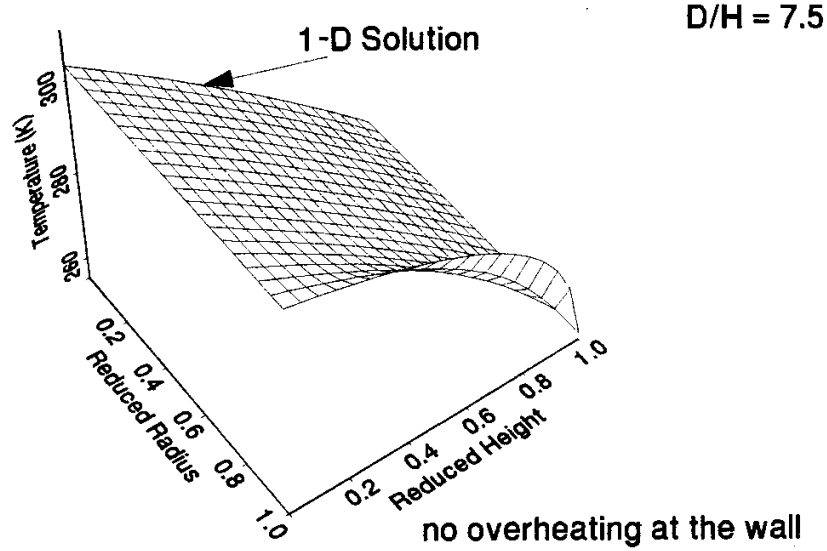

FIG. 1. Two-dimensional temperature profile for an experiment involving 1-propanol and helium with a dry chamber wall and no overheating at the wall. Also included is the comparison with the one-dimensional solution in the center.

fraction profile is relatively uniform, the energy balance is solved first, using the analytical solution for the mole fraction as the initial guess. Then, the mass balance is solved using the improved temperature profile. In the case of a wet wall, the order is reversed. In this case, the mass balance is solved first, since the radial temperature profile is relatively uniform. Then, the calculated mole fraction profile is used in the energy balance to improve the temperature profile. The process is repeated until the both the mole fraction and temperature profiles converge (usually achieved within four or so iterations). This is due in large part to the rather good initial guesses for the mole fraction or temperature profile. Once the mole fraction and temperature profiles are known, the supersaturation, nucleation rate, and density profiles are computed.

\section{RESULTS AND DISCUSSION}

The two-dimensional transport equations have been solved for a number of different experimental conditions. The data used for the temperatures and pressures in these simulations corresponds to actual experimental results obtained in our laboratory. We chose the 1-propanol/helium system for most of our simulations. This system has been studied extensively so there is data available for use in our simulations. ${ }^{1,2,4}$ Additional calculations were also made for 1-butanol with different background gases. ${ }^{3}$ These experiments (with 1-butanol) were carried out at higher temperatures and total pressures with a wet wall.

\section{Supersaturation and rate profiles}

Figures 1-4 show representative three-dimensional perspective plots of the temperature and mole fraction profiles that were obtained as numerical solutions to the twodimensional mass and energy balances [Eqs. (5) and (17)]. Figure 1 represents the temperature profile inside the HPCC 


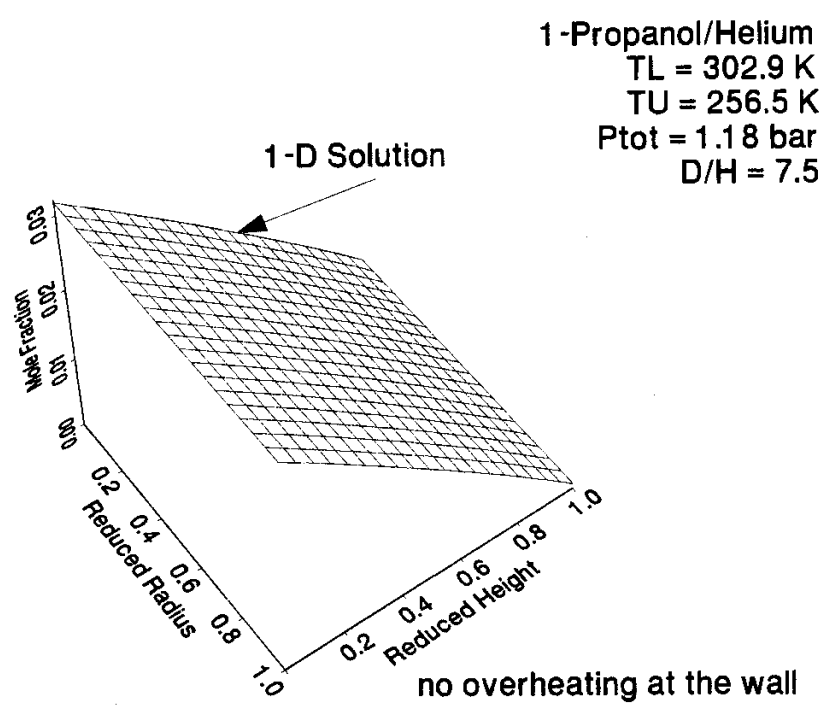

FIG. 2. Two-dimensional mole fraction profile for an experiment involving 1-propanol and helium with a dry chamber wall and no overheating at the wall. Also included is the comparison with the one-dimensional solution in the center. Since the vapor does not condense at the wall, the mole fraction profile is radially uniform.

for the case of a dry wall for an experiment involving 1-propanol in helium at a total pressure of 1.18 bar and lower and upper chamber plate surface temperatures of 302.9 and $256.5 \mathrm{~K}$, respectively. A brief description of this representative figure will aid in understanding the subsequent threedimensional perspective plots that also illustrate solutions to Eqs. (5) and (17).

The aspect ratio for the HPCC used in this experiment is 7.5 , and the wall was dry during the experiment. The temperature is plotted on the $z$ or vertical axis, and the $x y$ plane corresponds to the vertical cross section of the HPCC

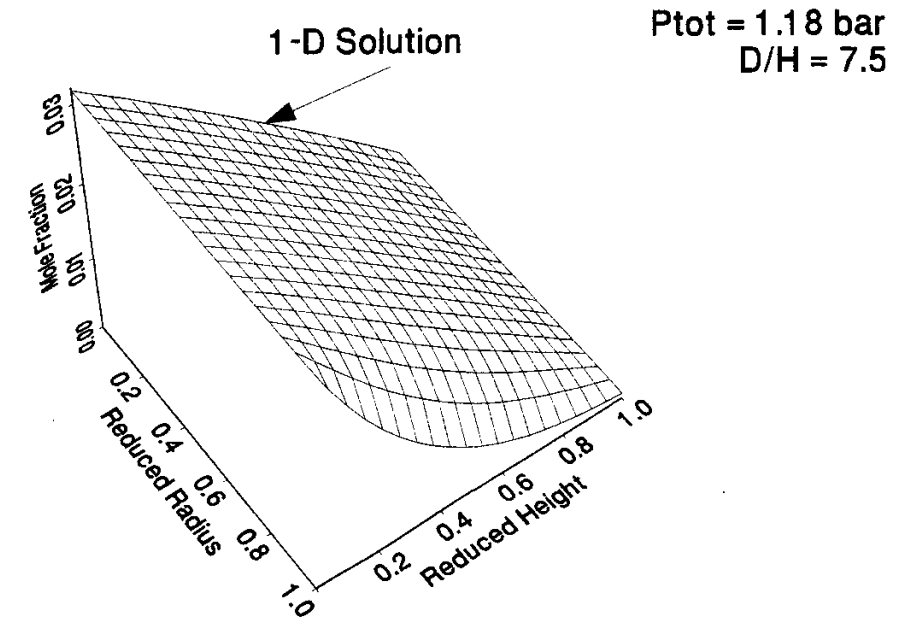

FIG. 3. Two-dimensional mole fraction profile for an experiment involving 1-propanol and helium with a wet chamber wall. Also included is the comparison with the one-dimensional solution in the center. The vapor condenses at the wall and is depleted in the outer region of the chamber.

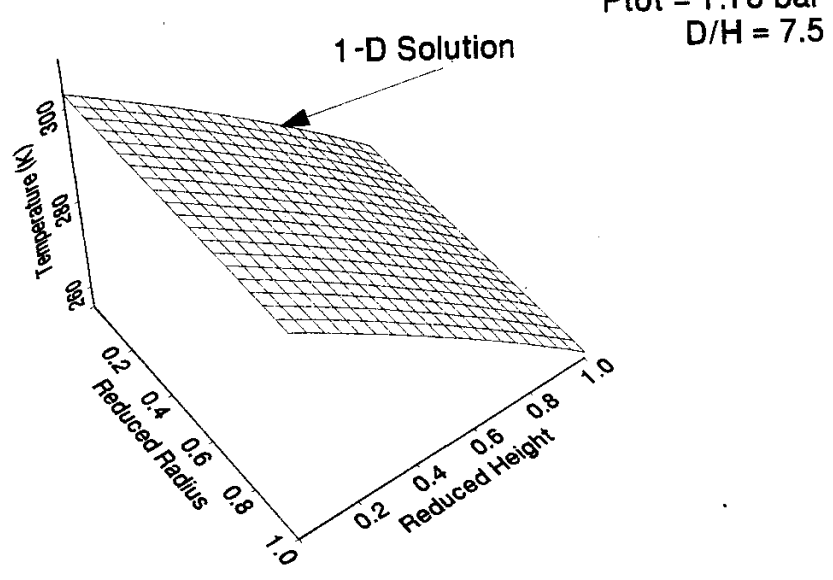

FIG. 4. Two-dimensional temperature profile for an experiment involving 1-propanol and helium with a wet chamber wall. Also included is the comparison with the one-dimensional solution in the center. The temperature profile is radially uniform.

(through the center). In this figure, the center of the HPCC begins at the base of the temperature axis and runs parallel to the reduced height axis. The Reduced Radius axis begins at the center of the HPCC and ends at the wall. The front of the diagram (parallel to the Reduced Radius axis) represents the conditions at the lower plate, the back of the diagram corresponds to the upper plate surface. We note from the figure that the temperature near the wall (shown along the Reduced Height axis) is elevated. The one-dimensional solution for the temperature profile is included in the plot to provide a comparison between the one- and two-dimensional solutions.

\section{Interpretation of the boundary conditions}

The primary difference between the wet and dry wall solutions, as reflected in the boundary conditions, is that in the wet wall case the temperature profile is uniform and the mole fraction profile is modified at the wall; while, in the dry wall case, the mole fraction profile is uniform and the temperature profile is modified at the wall. This is illustrated in Figs. 1-4, which show results for temperature and mole fraction profiles. The corresponding supersaturation and nucleation rate profiles are shown in Figs. 5 and 6.

Figure 1 illustrates the temperature profile in the dry wall case. In the center, the profile is nearly linear and agrees well with the one-dimensional solution. Toward the wall the temperature is elevated due to the heating of the wall. This increases the equilibrium vapor pressure near the wall, thus preventing condensation. In this simulation the wall was heated uniformly and just enough to prevent condensation. The partial pressure of the condensable is equal to the equilibrium vapor pressure, and the supersaturation along the wall is unity. Figure 2 shows the corresponding mole fraction profile. It is radially uniform and not affected by the wall. 


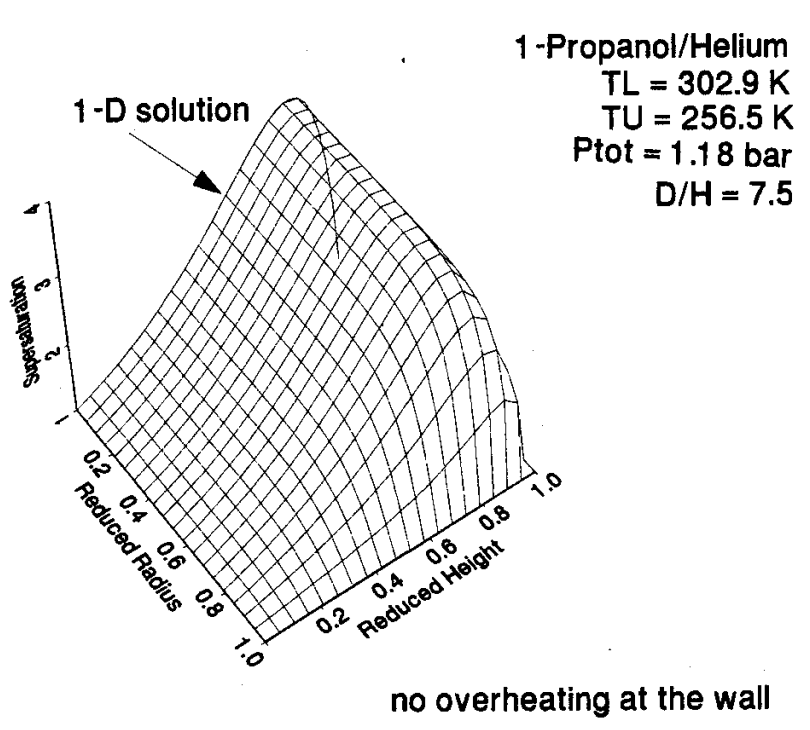

FIG. 5. Two-dimensional supersaturation profile for an experiment involving 1-propanol and helium with a dry chamber wall and no overheating at the wall. Also included is the comparison with the one-dimensional solution in the center

Figure 3 depicts the mole fraction profile in the wet wall case. It is no longer radially uniform but decreases sharply near the wall. This decrease is caused by condensation at the wall that depletes the vapor. The mole fraction is lowered until the partial pressure of the vapor is equal to the equilibrium vapor pressure resulting in a supersaturation of unity. The temperature profile, which is shown in Fig. 4, is radially uniform and not affected by the wall.

Figure 5 shows the corresponding three-dimensional perspective plot of the supersaturation profile inside the HPCC for the case of a dry wall. We note from the figure that the supersaturation falls to unity along the bottom and top plate

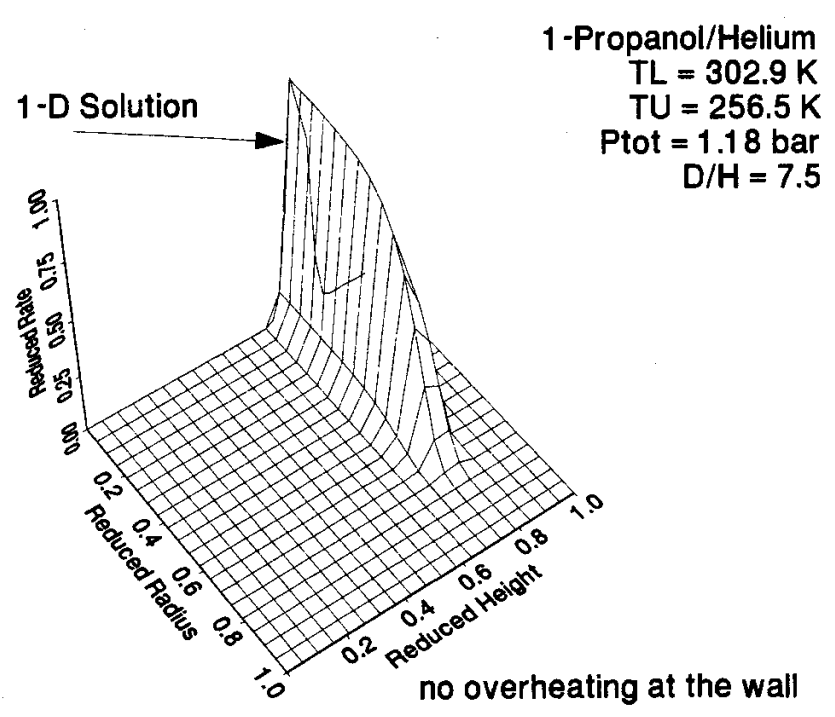

FIG. 6. Two-dimensional nucleation rate profile for an experiment involving 1-propanol and helium with a dry chamber wall and no overheating at the wall. Also included is the comparison with the one-dimensional solution in the center. The rate is normalized such that the maximum rate of the onedimensional solution is unity.

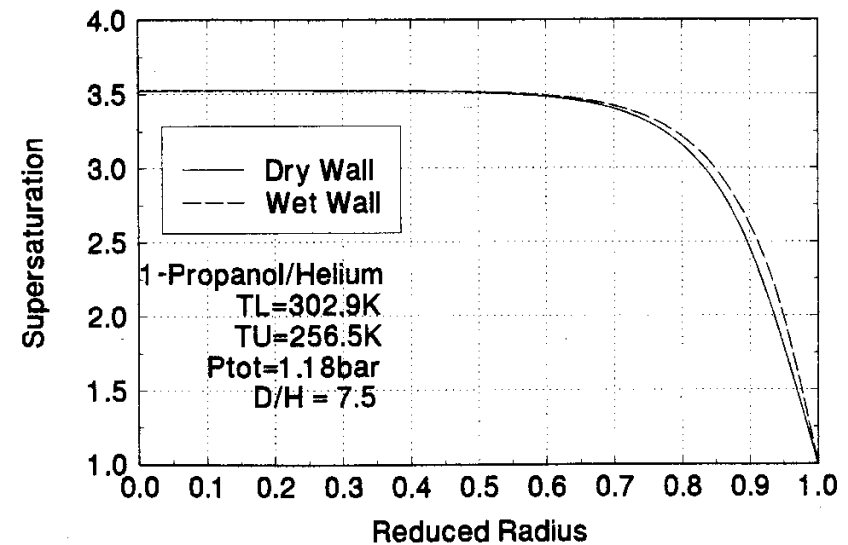

FIG. 7. Comparison between the radial supersaturation profile in the nucleation plane for a wet and dry chamber wall for an experiment involving 1-propanol and helium.

surfaces, as well as along the HPCC wall (shown along the Reduced Height axis). The largest values for the supersaturation are achieved in the center portion of the HPCC. The one-dimensional solution for the supersaturation profile is also included to provide a comparison between the one- and two-dimensional solutions. The supersaturation profile at the center of the HPCC agrees well with the one-dimensional solution, indicating that conditions at the center of the chamber in this calculation are not affected by the wall.

Figure 6 shows the plot for the nucleation rate for this experiment. The rate profile has been computed using the temperature and supersaturation profiles shown in Figs. 1 and 5 and the BDZ nucleation theory. The calculated rate has been normalized so that the maximum rate in the onedimensional case is unity and thus corresponds approximately to the rate observed in the experiment. Again, the one-dimensional solution has been included for comparison. The rate profile in the center of the HPCC agrees well with the one-dimensional solution, indicating that the center of the chamber is not affected by the wall under these conditions. The nucleation rate profile drops to zero in the vicinity of the bottom and top plate and close to the wall. It passes through a maximum at approximately, $75 \%$ of the height of the HPCC.

\section{Comparison of the wet and dry wall supersaturation and rate profiles}

In order to compare the effects of the behavior of the mole fraction and temperature profiles in the wet and dry wall cases, we compare the radial supersaturation and nucleation rate profiles for both cases. We focus on the height in the chamber at which the nucleation rate reaches a maximum - we refer to this height as the nucleation planesince this allows the use of two-dimensional plots and facilitates our comparison.

Figure 7 shows the radial dependence of the supersaturation in the nucleation plane for wet and dry walls. The supersaturation reaches the highest value at the center of the chamber and drops to unity at the wall. The radial supersaturation profiles for the wet and dry wall solutions are similar. 


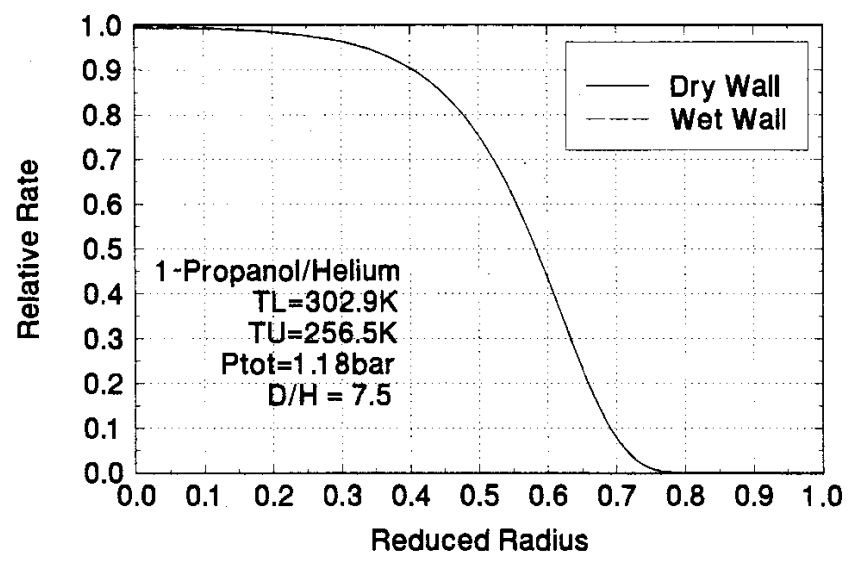

FIG. 8. Comparison between the radial nucleation rate profile in the nucleation plane for a wet and dry chamber wall for an experiment involving 1-propanol and helium. The nucleation rate at the center of the chamber reaches the value of the one-dimensional solution (indicated by a relative rate of unity).

The same is true for the nucleation rate profiles shown in Fig. 8. The same behavior has also been observed in our simulations involving 1-butanol at higher temperatures and pressures. The increase in the rate from zero to unity occurs at approximately the same radial position. The radial rate profile is particularly useful when discussing the influence of the chamber wall, since the rate is particularly sensitive to minor changes in the supersaturation. Recall that a reduced rate of unity indicates that the rate profile at that radial position is the same as the one computed using the onedimensional solution for which wall effects are not manifested. Thus, the disturbance in the temperature profile in the dry wall case has the same effect on the nucleation rate inside the chamber as the depletion of the vapor adjacent to the wall in the wet wall case. As long as the diameter-to-height ratio is sufficiently large, the influence of the walls is confined to the outer region and the mode of operation (wet or dry wall) does not affect nucleation measurements made in the central portion of the diffusion cloud chamber. Simulations of several experimentally obtained data points with varying temperature ranges and varying total pressure have shown that the influences of temperature and total pressure on the radial rate profile are virtually nonexistent.

\section{Aspect ratio study}

An important criterion for proper cloud chamber operation is the aspect (diameter to height) ratio. The larger the aspect ratio, the less the wall boundary conditions influence the central region of the chamber. For "sufficiently" large aspect ratios, the mass and energy transport in the center of the chamber is (essentially) one dimensional, and wall effects can be ignored. As stated earlier, an aspect ratio of 5 or greater has been regarded as "sufficiently large." The aspect ratio of the HPCC in which most of our recent experiments were performed is 7.5. To examine in a somewhat more quantitative fashion the influence of the aspect ratio, we have simulated cloud chamber operation for aspect ratios of $2.5,5$, 7.5 , and 10 .

Figure 9 shows the radial dependence of the supersatu-

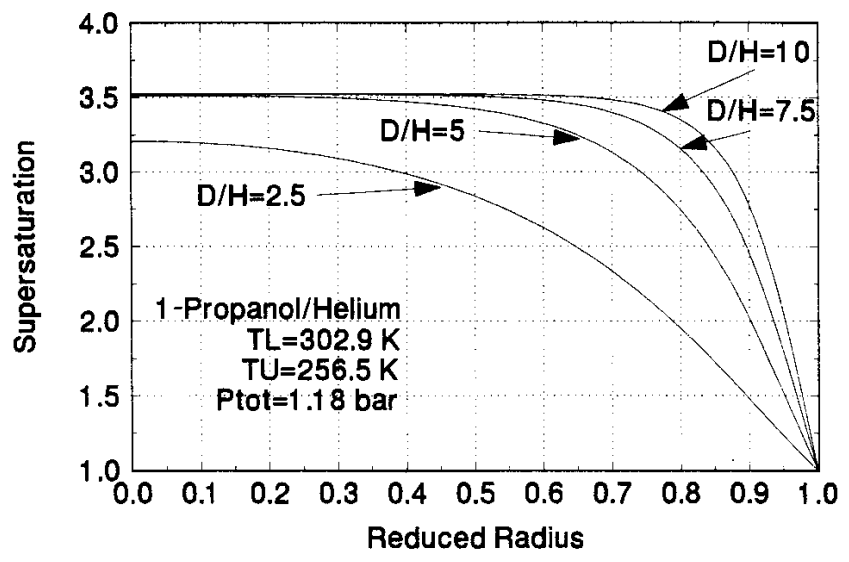

FIG. 9. Influence of the aspect (diameter to height) ratio on the radial supersaturation profile in the nucleation plane for a dry chamber wall for an experiment of 1-propanol in helium.

ration in the nucleation plane for these four aspect ratios, assuming a wet wall. The results for the dry wall calculations are virtually indistinguishable from the wet wall solutions (as has been pointed out previously). For aspect ratios of 5 and above the supersaturation at the center appears to reach the value obtained using the one-dimensional solution. For an aspect ratio of 2.5 , however, the supersaturation in the center is lower, indicating that the influence of the wall extends to the center of the chamber.

Figure 10 depicts the dependence of the nucleation rate in the nucleation plane on radial position. For aspect ratios of 7.5 and 10 the nucleation rate in the center of the chamber is equal to that of the one-dimensional solution. We note, however, that for an aspect ratio of 5 , the nucleation rate in the center is decreased due to the influence of the wall. Even though the supersaturation for this aspect ratio was essentially that of the one-dimensional value in the center, the rate is approximately $15 \%$ lower and drops off quickly with increasing radius. This, of course, is a manifestation of the strong dependence of the rate on the supersaturation. For an

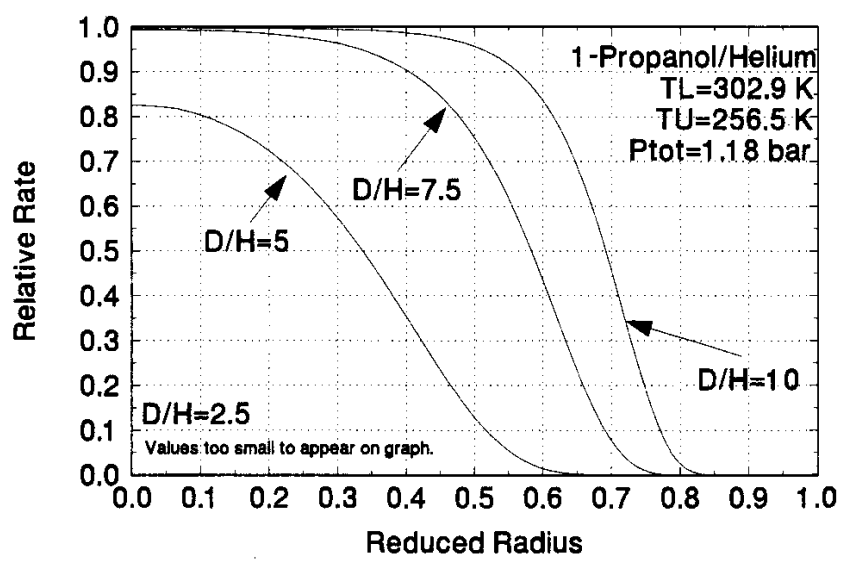

FIG. 10. Influence of the aspect (diameter to height) ratio on the radial nucleation rate profile in the nucleation plane for a dry chamber wall for an experiment of 1-propanol in helium. 
aspect ratio of 2.5 the nucleation rate is dramatically reduced throughout the chamber.

The results of these aspect ratio studies strongly suggest that it is important to consider the radial dependence of the nucleation rate before attempting quantitative nucleation rate measurements. There must not be a significant variation in rate in the region over which the rate is being measured unless that variation is accounted for: As can be seen in Fig. 10 , for an aspect ratio of 5 and an observation region extending to a reduced radius of 0.5 , the rate obtained from our two-dimensional calculation is roughly one-half that obtained using the one-dimensional solution. Even for an aspect ratio of 7.5 , for which the rate in the center agrees with the one-dimensional solution, the rate measurement must to be limited to a region extending approximately one-third of the reduced radius from the center, where the reduced rate is essentially unity. This is a particularly important point to consider when making nucleation measurements at low nucleation rates, e.g. $10^{-1} \mathrm{drops} / \mathrm{cm}^{3} / \mathrm{s}$ and less, since the tendency often is to measure the rate over a wider area within the chamber.

\section{Effect of overheating the wall}

In the case of a dry wall, the temperature at the wall has to be just high enough to prevent condensation. However, as mentioned during our discussion of the boundary conditions, in actual experiments the temperature at the wall is often elevated beyond this level, resulting in supersaturations of less than unity at the wall. This would occur, for example, when two or three heating wires are used to clear the entire wall. In our analysis, we model this by introducing an overheating function, $\mathcal{X}(z)$ [see Eq. (22)], that depends upon reduced height. This function is used to compute the wall temperature as follows:

$$
\theta^{b c}=\theta^{\mathrm{lin}}+\gamma(z) \cdot\left(\theta^{\mathrm{eq}}-\theta^{\mathrm{lin}}\right),
$$

where $\theta^{b c}$ is the dimensionless temperature at the wall, $\theta^{\mathrm{eq}}$ is the dimensionless temperature for which the supersaturation is unity, and $\theta^{\text {in }}$ corresponds to the dimensionless temperature according to a linear temperature profile. This calculation of the temperature at the wall ensures that the temperatures at the top and the bottom of the wall are equal to the temperatures of the respective plates, thus avoiding singularities in the boundary conditions. A value of $\gamma=1$ corresponds to no overheating. The function $\gamma(z)$ given as

$$
\gamma(z)=1+g \cdot g_{2} \cdot \frac{1}{2} \cdot\left[1-\cos \left(2 \pi g_{3} z\right)\right],
$$

where $g, g_{2}$, and $g_{3}$ are parameters that can be adjusted to tailor the temperature profile along the chamber wall. The $g$ term provides even overheating, and the $g_{2}$ term will produce a periodic overheating with $g_{3}$ representing the number of heating wires being used.

Figure 11 depicts the radial dependence of the supersaturation in the nucleation plane in the case of even overheating. We note that the higher the degree of overheat, the lower the supersaturation at the wall. These low values of the supersaturation propagate into the chamber. The effect on the radial nucleation rate profile is shown in Fig. 12. For higher

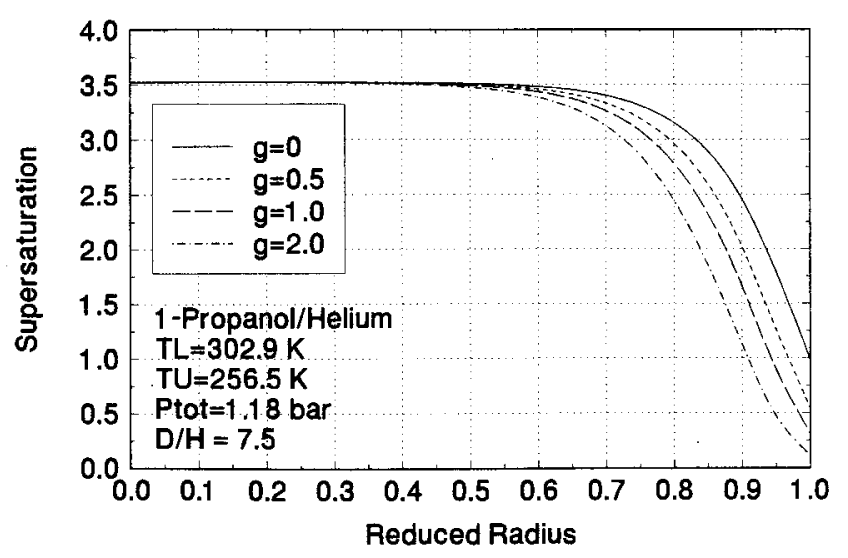

FIG. 11. Influence of overheating at the dry wall on the radial supersaturation profile in the nucleation plane for an experiment involving 1-propanol and helium. If the chamber wall is overheated, the supersaturation drops below unity at the wall.

degrees of overheat the effect (decrease) on the nucleation rate moves closer to the center. We note, however, that for the results shown in Fig. 12, the rate at the center of the chamber is not significantly affected. It is important to note that for these simulations, a degree of overheat of 2.0 corresponds to an increase in wall temperature at the height of the nucleation plane of approximately $35 \mathrm{~K}$ beyond the temperature required to dry the walls. Thus, transport effects as modeled with the mass and energy balance equations, are not sufficient to explain the worrisome dependence of nucleation rate on the wall heat, which has been observed experimentally. ${ }^{12}$

In actual operation, the diffusion cloud chamber wall (e.g., the quartz or Pyrex ring) is usually heated using two to four heating wires. ${ }^{14,15}$ Because of the discrete location of the heating wires, even heating of the wall is not possible. In order to prevent condensation in the cooler areas between the heating wires, the regions in the vicinity of the heating wires are overheated. Figure 13 depicts the temperature profile near the wall for the case of three heating wires. The case of

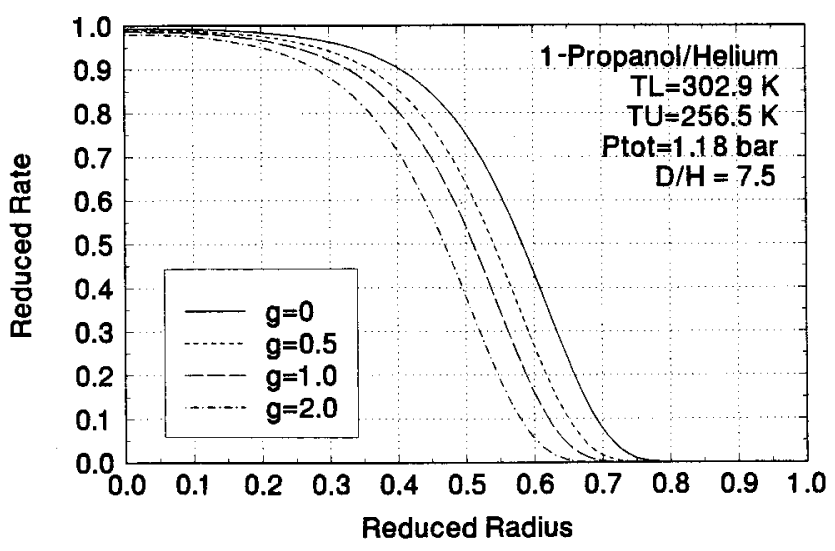

FIG. 12. Influence of overheating at the dry wall on the radial nucleation rate profile in the nucleation plane for an experiment involving 1-propanol and helium. The rate at the center of the chamber reaches unity in all cases and thus is the same as that computed from the one-dimensional solution. 


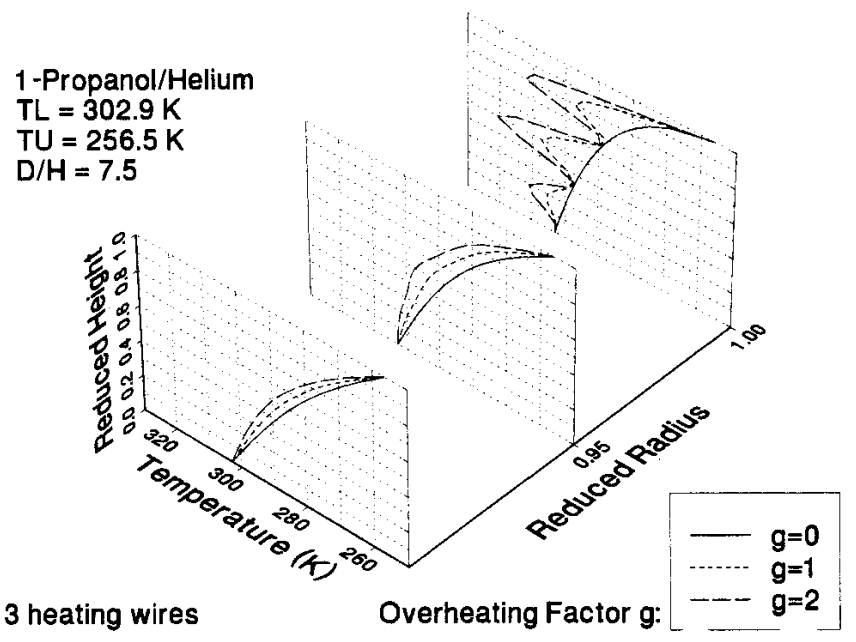

FIG. 13. Temperature profiles near the wall (at reduced radii of $0.9,0.95$, and 1.0) for a dry chamber wall and an experiment involving 1-propanol and helium. The chamber wall is heated by three heating wires, resulting in a periodic temperature profile at the wall.

no overheating (uniform heating) is represented by a solid line. At the wall the sinusoidal temperature profile caused by the three heating wires is clearly seen. Interestingly, these oscillations damp out rather quickly; in fact, at a reduced radius of 0.95 they are nearly undetectable. Thus, the effect of overheating on the supersaturation and nucleation rate profiles in the chamber tends to be the same for even, as well as periodic, overheating. The differences between the two kinds of wall heating are confined to a region close to the wall.

\section{Effect of the background gas on the one-dimensional supersaturation and rate profiles}

Using different background gases affects the shape of the temperature and mole fraction profiles and thus changes the shapes of the supersaturation and rate profiles. We have observed experimentally that an increase in total pressure shifts the position of the (observable) nucleation plane toward the upper plate. Furthermore, we have observed experimentally that using a more dense background gases also shifts the nucleation plane toward the upper plate. ${ }^{3,19}$

Here we compare the temperature, supersaturation, and nucleation rate profiles for experiments involving 1-butanol in hydrogen, helium, nitrogen, and argon. These are computed profiles based on actual experiments. The total pressure for the experiments in hydrogen and helium was 7.5 bar; for nitrogen, $3.9 \mathrm{bar}$; and for argon, 2.8 bar. During these experiments, the temperatures at the top and bottom plate were adjusted so that the observed rate was $2-4 \mathrm{drops} / \mathrm{cm}^{3} / \mathrm{s}$ and the calculated nucleation temperature was approximately $334 \mathrm{~K}$ for all four experiments.

Figure 14 depicts the temperature profiles at the center of the chamber for the four different background gases. The profiles for hydrogen and helium are nearly linear, while the profiles for nitrogen and argon are more curved. The different thermodynamic properties required for the solution of the

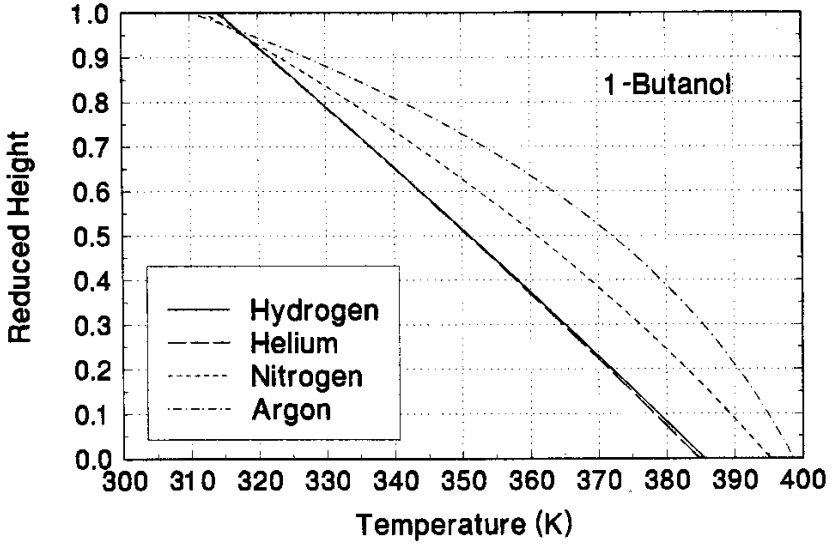

FIG. 14. One-dimensional temperature profiles in the center of the chamber for experiments with 1-butanol in four different background gases.

mass and energy transport equations are included in the dimensionless Lewis number:

$$
\mathrm{Le}=\frac{\lambda}{c D_{12} \cdot c_{p}} .
$$

The mixture of 1-butanol/ $/ \mathrm{H}_{2}$ has the highest Lewis number $(\mathrm{Le}=0.7935$ at the lower plate $), 1$-butanol/Ar the lowest (Le $=0.3882$ at the lower plate). Thus, for the heavier gases the convective transport plays a more important role, and the resulting temperature profiles differ significantly from the common but simplifying assumption of a linear profile.

This difference in the temperature profiles affects the supersaturation profiles. Figure 15 depicts the supersaturation profiles in the center of the chamber for the four different background gases. The location of the maximum supersaturation shifts upward for the heavier background gases. These changes in the temperature and supersaturation profiles result in an upward shift of the nucleation plane, as is shown in Fig. 16, where we have plotted the four reduced (compared to the one-dimensional value) nucleation rate profiles at the center of the chamber. For 1-butanol in hydrogen

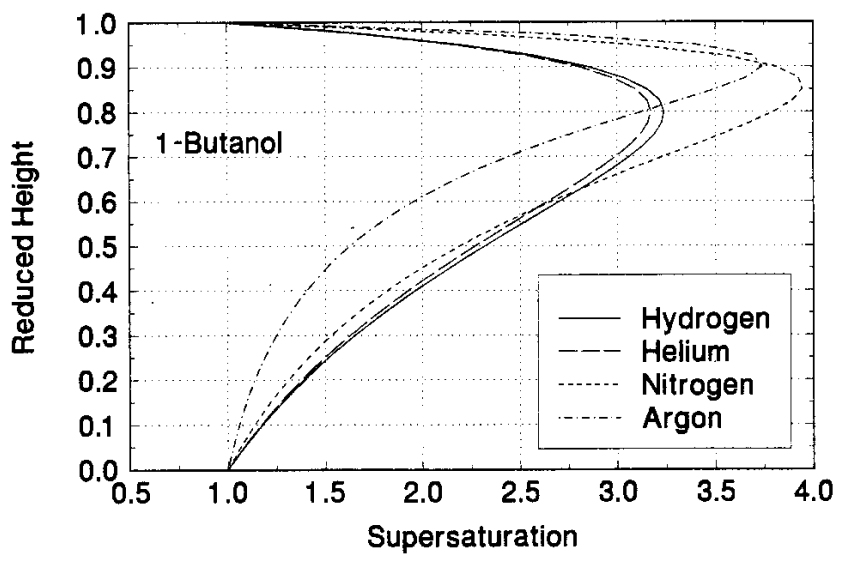

FIG. 15. One-dimensional supersaturation profiles in the center of the chamber for experiments with 1-butanol in four different background gases. 


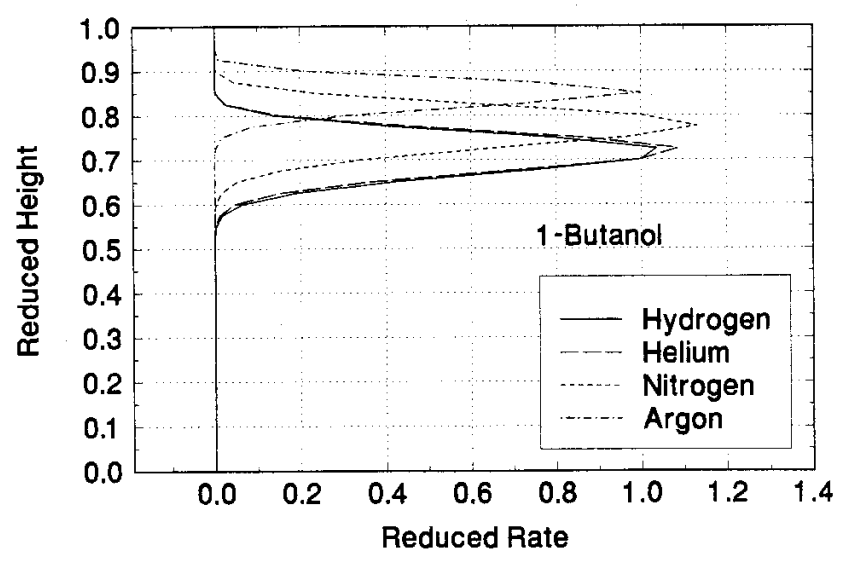

FIG. 16. One-dimensional nucleation rate profiles in the center of the chamber for experiments with 1-butanol in four different background gases.

or helium the rate reaches its maximum at a reduced height of 0.7 ; for nitrogen the reduced height shifts to 0.75 ; and for argon it shifts to 0.85 .

This shift in height is entirely consistent with our experimental observations. ${ }^{3}$ This shift in position of the nucleation plane toward the cooler plate surface could present a significant difficulty in the design of a downward diffusion cloud chamber. In such a chamber the vapor diffuses downward from the hot, upper plate to a cooler, lower plate. For some time the downward diffusion cloud chamber has been regarded as a possible solution to the buoyancy-driven convection limitations inherent to the upward diffusion cloud chamber when working with heavy background gases at elevated pressures. Apart from the difficulty of maintaining a liquid pool at the upper plate, the nucleation plane will move closer to the bottom plate, leaving only small distances over which the droplets form and fall. This behavior will make the detection of nucleation and the measurement of nucleation rate difficult.

\section{SUMMARY AND CONCLUSIONS}

We have defined and solved two-dimensional mass and energy transport equations describing the operating conditions at every point in the diffusion cloud chamber. We have introduced wet and dry conditions at the wall through the use of appropriate boundary conditions. Several key factors were examined for their influence on the shape of supersaturation and nucleation rate profiles throughout the diffusion cloud chamber.

The aspect ratio of the diffusion cloud chamber is an important design criterion since an inappropriate choice can seriously compromise proper operation of the chamber and deleteriously affect the results of nucleation measurements. While in the past this was an empirically based design decision, here we present a quantitative criterion for a choice of an appropriate value. We have shown that for typical vaporgas systems, such as those examined in this paper, an aspect ratio of less than approximately 7.5 can lead to questionable results if the nucleation rate is measured over too large an area within the chamber, and this area of measurement is not properly considered in the analysis of the nucleation rate data. The measured nucleation rate, while seemingly steady and reproducible, can easily be in error by a factor of 2 or more. With the emphasis currently on quantitative nucleation rate measurements, it is important that these sorts of (correctable) measurement errors be properly accounted for. For the measurement of onset of nucleation (critical supersaturation), it is possible to use aspect ratios of 5 or greater.

From our model calculations, we conclude that the presence of a wet or dry wall does not seriously affect the interior conditions of the diffusion cloud chamber. Operation with either a wet or dry wall is permitted provided the (dry) wall is not overheated. The supersaturation and temperature conditions in the center (roughly) three-quarter (by volume) region of the chamber remain, essentially, unaffected by having the chamber wall wet or dry. The effect of the wall does not propagate into the center region of the chamber. However, this is no longer the case if the (dry) wall is overheated. Significant overheating of the wall leads to deleterious effects on the nucleation rate profile in the center portion of the cloud chamber. We also find that spatial variations of the wall temperature (due to the presence of heater wires along the outside surface of the chamber ring) damp out rapidly and do not propagate far into the chamber. In this case, while overheating can still be a problem, the spatial variation of the heater wires is not. Considering the potential deleterious effect of wall (over)heating, it is recommended that diffusion cloud chambers be operated in the wet wall mode when convenient. If not, then special attention must be paid to the wall heating. This is an important conclusion and addresses, in a positive fashion, a significant concern regarding reliable operation of the HPCC with a thick, wet quartz wall.

Our two-dimensional model calculations predict background gas effects on the location of the supersaturation and nucleation rate profiles within the cloud chamber that agree well with our experimental observations. We observe that as the density of the background gas increases or the total pressure increases, the height of the nucleation plate increases (moves toward the upper plate), in complete agreement with our model calculations. This suggests that operation of the diffusion cloud chamber in the downward mode will not be an effective approach to investigating nucleation in the presence of more dense background gases or at higher total pressures.

Finally, one additional comment needs to be made regarding the effects of wall temperature and background gas on the operation of the diffusion cloud chamber. An important consideration for proper diffusion cloud chamber operation is the stability (with respect to buoyancy-driven convective motion) of the vapor-gas mixture within the chamber. The density gradient in the interior portion of the chamber must decrease monotonically with height; even small local inversions may propagate and lead to unstable behavior, resulting in convective disturbances (often clearly visible due to the increased rates of nucleation and/or the entrained nucleated droplets scattering light as they fall with curved trajectories), precluding reliable nucleation measurements. A second, less obvious but potentially more damaging, stability 
consideration concerns local inversions of the vapor-gas mixture density gradient adjacent to the chamber wall that can cause subtle, convective flows that may be quite difficult to detect but that can have a profound influence on the measured nucleation rate in the center portion of the chamber. Local density inversions near the wall can occur for both wet and dry wall operation. Vapor-gas mixture stability (with respect to buoyancy-driven convection) is, perhaps, one of the more important issues facing investigators using diffusion cloud chambers. Our two-dimensional analysis of diffusion cloud chamber operation has shed new light on our understanding of these kinds of effects, and we address them in detail in a following paper.

\section{ACKNOWLEDGMENTS}

We acknowledge helpful discussions with Ye Peng, Shenbo Yu, and Donald He, and support by the Department of Chemical Engineering at the University of Rochester.

\section{APPENDIX}

\section{Cloud chamber ring condensate film thickness}

In the case of wet walls where the working fluid vapor condenses on the quartz ring, the thickness of the liquid film can be estimated. The total mass flow rate at any given height $z$ can be obtained by integrating the mass flux to the wall:

$$
\begin{aligned}
Q(z) & =\int_{0}^{2 \pi} \int_{H}^{H-z} R \cdot \mathrm{MW} \cdot N_{r}(z, r=R) d z \\
& =2 \pi R L(z),
\end{aligned}
$$

where $L(z)$ is the cumulative mass flux and MW is the molecular weight of the condensable. The mass flow rate can be calculated by numerically integrating the values for the radial flux next to the wall obtained from the mole fraction profile. The thickness of the film is assumed to be negligible compared to the radius of the chamber.

The total mass flow rate at any given height can also be obtained from the velocity profile. For the calculation of the velocity profile, a pseudolaminar flow is assumed. The velocities perpendicular to the wall are assumed to be negligible, the thickness of the film, however, depends on the height. Furthermore, the curvature is neglected since the thickness of the film is much smaller than the radius of the chamber. This allows the use of Cartesian coordinates, which simplifies the expression for the wall thickness.

The Navier-Stokes equation for the laminar flow of a Newtonian fluid down a vertical wall is given as

$$
\rho g+\mu \frac{\partial^{2} \nu_{z}}{\partial x^{2}}=0
$$

where $x$ is the direction perpendicular to the wall, $\rho$ is the density, $g$ is the acceleration due to gravity, $\mu$ is the liquid viscosity, and $v_{z}$ is the velocity of the film down the wall.

The boundary conditions are no slip at the wall and a free surface (extra stress tensor vanishes) at the liquid-gas interface:

$$
\begin{aligned}
& \nu_{z}(x=0)=0, \\
& \left.\frac{d \nu_{z}}{d x}\right|_{x=\delta(z)}=0,
\end{aligned}
$$

where $\delta(z)$ is the height dependent thickness of the film. The velocity profile is given by

$$
\nu_{z}(x)=\frac{\rho g}{2 \mu} \delta^{2}\left(\frac{2 x}{\delta}-\frac{x^{2}}{\delta^{2}}\right),
$$

where $x$ corresponds to $(R-r)$.

The total mass flow rate at any given height $z$ is obtained by integrating the velocity profile:

$$
Q(z)=\rho \cdot \dot{V}(z)=2 \pi R \rho \int_{0}^{\delta(z)} \nu_{z}(x) d x=\frac{2 \pi R \rho^{2} g}{3 \mu} \delta^{3}(z),
$$

where $\dot{V}(z)$ is the volumetric flow rate. By equating the two expressions for the total mass flow rate [Eqs. (A1) and (A5)], we obtain an equation for the film thickness as a function of height:

$$
\delta(z)=\sqrt[3]{\frac{3 \mu L(z)}{\rho^{2} g}} .
$$

The average falling velocity of the film can be computed from the volumetric flow rate:

$$
\left\langle\nu_{z}(z)\right\rangle=\frac{2}{3} \nu_{\max }(z)=\frac{\rho g \delta^{2}}{3 \mu} .
$$

\section{LIST OF SYMBOLS}

$r$

$R$

$z$

$H$

$N$

$q$

$x$

$T$

$c$

$D_{A B}$

$b$

$\bar{H}$

$c_{p}$

$\lambda$

Le

$\alpha_{T}$

$T_{L}$

$T_{U}$

$x_{L}$ radial coordinate

chamber radius

axial coordinate

chamber height

molar flux

heat flux

mole fraction of the condensable in the gas phase temperature

concentration

binary diffusion coefficient

exponent for the temperature dependence of the diffusion coefficient

enthalpy of the vapor

heat capacity of the vapor

thermal conductivity

Lewis-number

thermal diffusion coefficient

temperature of the lower plate

temperature of the upper plate

mole fraction of the condensable at the lower plate mole fraction of the condensable at the upper plate slope of the linear temperature profile total pressure 
$p^{\text {eq }} \quad$ equilibrium vapor pressure

$\gamma \quad$ overheating function at the wall

$R \quad$ universal gas constant

$\eta \quad$ dimensionless radius

$\xi \quad$ dimensionless height

$\theta$ dimensionless temperature

$\psi$ dimensionless expression for the mole fraction: $\psi=\ln (1-x)$

\section{Film thickness calculation (Appendix)}

$Q \quad$ mass flux towards the wall

$L \quad$ cumulative flux down the wall

MW molecular weight

$\rho$ density

$\mu \quad$ liquid viscosity

$g \quad$ gravitational constant

$v \quad$ velocity of the fluid down the wall

$\delta \quad$ thickness of the film at the wall

$x \quad$ coordinate perpendicular to the wall

$V \quad$ volumetric flow rate

${ }^{1}$ R. H. Heist, M. Janjua, and J. Ahmed, J. Phys. Chem. 98, 4443 (1994).

${ }^{2}$ R. H. Heist, J. Ahmed, and M. Janjua, J. Phys. Chem. 99, 375 (1995).

${ }^{3}$ A. Bertelsmann, R. Stuczynski, and R. H. Heist, J. Phys. Chem. 100, 9762 (1996); A. Bertelsmann, Ph.D. thesis, University of Rochester, 1997.

${ }^{4}$ R. H. Heist, J. Phys. Chem. 99, 16792 (1995).

${ }^{5}$ V. Vohra and R. H. Heist, J. Chem. Phys. 104, 382 (1996); V. Vohra, Ph.D. thesis, University of Rochester, 1996.

${ }^{6}$ R. H. Heist, in Physical Chemistry of Aqueous Systems-Meeting the Needs of Industry, Proceedings of the 12th International Conference on the Properties of Water and Steam, edited by H. J. White, Jr., J. V. Sen- gers, D. B. Neumann, and J. C. Bellows (Begell House, New York, 1995), pp. $286-293$.

${ }^{7}$ R. H. Heist and A. Bertelsmann, in Nucleation and Atmospheric Aerosols 1996, Proceedings of the 14th International Conference on Nucleation and Atmospheric Aerosols, edited by M. Kulmala and P. E. Wagner (Pergamon, Elsevier, London, 1996), pp. 69-72.

${ }^{8}$ See, for example, R. Strey, Y. Viisanen, and P. E. Wagner, Finnish Assoc. Aer. Res. Rep. Aer. Sci. 17, 79 (1991); P. E. Wagner, R. Strey, and Y. Viisanen, Nucleation and Atmospheric Aerosols, Proceedings of the 13th International Conference on Nucleation and Atmospheric Aerosols, edited by N. Fukuta and P. E. Wagner (Deepack, Hampton, VA, 1992), p. 27; Y. Viisanen, R. Strey, and H. Reiss, J. Chem. Phys. 99, 4680 (1993); G. Wilemski, B. E. Wyslovzil, M. Gauthier, and M. B. Frish, in Nucleation and Atmospheric Aerosols, Proceedings of the 13th International Conference on Nucleation and Atmospheric Aerosols, edited by N. Fukuta and P. E. Wagner (Deepak, Hampton, VA, 1992), p. 23; J. Hruby, Y. Viisanen, and R. Strey, J. Chem. Phys. 104, 5181 (1996).

${ }^{9}$ I. J. Ford, J. Aero. Sci. 23, 447 (1992); I. J. Ford, in Ref. 7, p. 39

${ }^{10} \mathrm{D}$. Oxtoby (private communication).

${ }^{11}$ O. V. Vasil'ev and H. Reiss, submitted to Phys. Rev. E; O. V. Vasil'ev and H. Reiss, submitted to J. Chem. Phys.

${ }^{12}$ Reported at Nucleation Experiments: State of the Art and Future Developments, an international workshop held at the Czech Technical University, Prague, Czech Republic, June 1995; also, see J. A. Fisk, V. M. Chakarov, and J. L. Katz, J. Chem. Phys. 104, 8657 (1996)

${ }^{13}$ J. L. Katz and B. J. Ostermier, J. Chem. Phys. 47, 478 (1967).

${ }^{14}$ J. L. Katz, J. Chem. Phys. 52, 4733 (1970).

${ }^{15}$ R. H. Heist and H. Reiss, J. Chem. Phys. 59, 665 (1973).

${ }^{16}$ R. H. Heist, in Handbook for Heat and Mass Transfer Operations, edited by N. Cheremisinoff (Gulf, Houston, TX, 1985), Vol. 1, Chap. 19.

${ }^{17}$ R. B. Bird, W. E. Stewart, and E. N. Lightfoot, in Transport Phenomena (Wiley, New York, 1960), Chaps. 10 and 18.

${ }^{18}$ R. C. Reid, J. M. Prausnitz, and B. E. Poling in The Properties of Gases and Liquids (McGraw-Hill, New York, 1987), Chap. 11.

${ }^{19}$ A. Bertelsmann and R. H. Heist (unpublished). 